\title{
Piecemeal Organization and Cognitive Components in Object Perception: Perceptually Coupled Responses to Moving Objects
}

\author{
Julian Hochberg \\ Columbia University
}

\author{
Mary A. Peterson \\ State University of New York at Stony Brook
}

\begin{abstract}
In three experiments, observers who were instructed to perceive one of two alternative depth arrangements of a three-dimensional wire cube fixated near one of two intersections that differed in the degree to which they specified the cube's veridical depth organization. In order to separate perceptual effects from experimenter effects, we measured indirect reports about variables perceptually coupled to perceived depth rather than direct reports about perceived depth. In all three experiments, reversal durations at the two intersections differed, even though the two were parts of a single object. In addition, reversals varied with viewers' intentions. Thus, the unit of perceptual organization may be smaller than the entire object, and viewers' intentions can influence the perception of real moving objects. In additional analyses, reversal durations were separated into two components: nonelective instability and malleability: the question of whether these two components of ambiguity are functionally distinct could not be decided.
\end{abstract}

Some objects and events are patently ambiguous in that they change in appearance from time to time, even though the stimulus objects themselves remain unchanged. For example, the reversible Necker cube shown in Figure 1A can be seen in either one of the two organizations shown in Figures $1 \mathrm{~B}$ and 1C. Although ambiguity is often said to be confined to static line drawings and other artificially impoverished stimuli (e.g., Gibson, 1966, 1979; Michaels \& Carello, 1981), three-dimensional (3D) objects in motion can also be ambiguous (von Hornbostel, 1922; Peterson \& Hochberg, 1983).

The ambiguous 3D object used by Peterson and Hochberg (1983) was a cube similar to the one shown in Figure 1D. The cube was constructed of wire with a surface wrapped over part of the framework providing kinetic occlusion (Gibson, Kaplan, Reynolds, \& Wheeler, 1969; Kaplan, 1969) to reveal the cube's true depth organization. Despite the clearly indicated depth information given by the wrapped surface, however, observers reported seeing the cube in a reversed orientation for nontrivial proportions of the viewing time (30-s trials). Observers reported more reversals when they were instructed to fixate a region of the cube near which no surface supplemented the depth cues given by the wires themselves (i.e., the unbiased intersection, 2, in Figure 1D), than when they looked near the surface information (i.e., near the biased intersection, 1, in Figure 1D; Peterson \& Hochberg, 1983). No part of the stimulus object used in these experiments fell far from the fovea; the distance between the

This research was supported by National Science Foundation Grant BNS 82-09470 to Julian Hochberg, and by a Public Health Service Biomedical Research Support Grant (2S07RR0706721) to Mary Peterson.

We thank Roy Galifi for help in conducting Experiment 2.

Correspondence concerning this article should be addressed to Julian Hochberg, Department of Psychology, Columbia University, New York, New York 10027; or to Mary A. Peterson, Department of Psychology, State University of New York, Stony Brook, New York 11794-2500. two fixation points was approximately $1.8^{\circ}$ of visual angle. Nevertheless, the effects of the instructions to attend to one or the other intersection suggest that the information at the biased intersection did not fully determine the organization perceived at the unbiased intersection.

In addition, Peterson and Hochberg (1983) manipulated viewers' perceptual intentions by asking them to try to maintain a local organization of the cube that was consistent with one of its alternative organizations. With these combined fixation and intention instructions, perceptual organization was found to be a function both of the viewer's intentions and of the local stimulus cues at the attended intersection. These results are important in evaluating several theories of perceptual organization.

Consider first those global simplicity theories (Hochberg, 1981) that predict perceptual organization by computing measures across the entire stimulus object (Attneave, 1954, 1972; Boselie \& Leeuwenberg, 1986; Buffart, Leeuwenberg, \& Restle, 1981; Butler, 1982; Hochberg \& Brooks, 1960; Hochberg \& MacAlister, 1953; Leeuwenberg, 1971). In such theories, the informational simplicity of each interpretation of a multistable figure is computed (coded) across the entire figure. The interpretation requiring the least coded information is predicted to be the preferred percept. These theories remain current (Hatfield \& Epstein, 1985) despite the fact that there has long been reason to question whether the parts of a perceived object are in any direct sense determined by the entire stimulus configuration or even need be consistent with each other (Dinnerstein \& Wertheimer, 1957; Hochberg, 1962, 1968; Penrose \& Penrose, 1958). Peterson and Hochberg's (1983) data argue strongly (a) that the determinants of organization can be computed across less than the entire object, and therefore that the organization for a single object may occur in a piecemeal fashion and (b) that perceptual organization can be influenced by the viewers' intentions. None of the global minimum principles 
has made explicit provisions for either of these variables, and it is not clear how they could do so (Hochberg, 1982, p. 212; Pomerantz \& Kubovy, 1986). ${ }^{1}$

Consider next those theories that explain perception in terms of a one-to-one mapping from higher order variables of stimulation without recourse to extrastimulus variables (e.g., Gibson, 1966; Johansson, 1982). Although they used somewhat different formulations, both Gibson and Johansson argued that an invariant or rigid object that is in motion relative to the viewer, providing suprathreshold parallax, is directly, unambiguously, and correctly perceived. However, piecemeal reversals of the moving 3D cube suggest that the stimulus information that is generated by motion is not automatically or unambiguously picked up, but is effective only when the viewer looks at or attends to the more informative parts of the object. Aside from the metatheoretical commitments of "direct" theorists to avoid "enrichment" through contributions by the perceiver, nothing inherent in those approaches prevents considering such extrastimulus variables. The effects of intention and fixation suggest that extrastimulus variables must be taken into account if we are to understand the conditions that permit nonambiguous perception.

Piecemeal instructed reversals also challenge those theories of attention that claim that the entire object is the unit of attention (Kahneman \& Henik, 1981; Kahneman \& Treisman, 1984).

Given the theoretical weight to be placed on piecemeal and elective reversal, the next question is whether these phenomena are truly perceptual, and not merely judgmental. That is, it is possible that viewers following intention instructions merely estimated the strength of the depth cues at each fixation point and interpreted those cues as covert instructions as to how to bias their responses. Some assurance that the results are truly perceptual, according to some consequential criterion, is necessary.

\section{Perceptually Coupled Responses}

The occurrence of perceptual couplings (Hochberg, 1956, 1974) offers a measurable criterion for saying that some response is perceptual rather than judgmental (Gogel \& Newton, 1976; Hochberg, 1974; Wallach, O'Connell, \& Neisser, 1953).

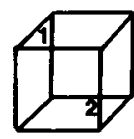

A

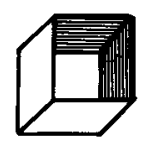

B

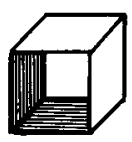

C

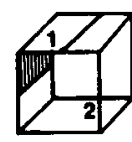

D

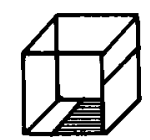

$\mathbf{E}$
Figure 1. Five cube presentations. (A: A reversible Necker cube. The intersections marked 1 and 2 correspond to the instructed fixation points used in Experiments 1-3. B: One alternative organization for Cube A. Experiment 2 used a cube biased at the top intersection toward this interpretation. C: A second alternate interpretation of Cube A. D: The cube used in Experiment 3. Fixation points are marked 1 and 2. Point 1 is the biased intersection and Point 2 is the unbiased intersection. E: The cube used in Experiment 1. Here the bottom intersection is biased, the top intersection remains unbiased.)

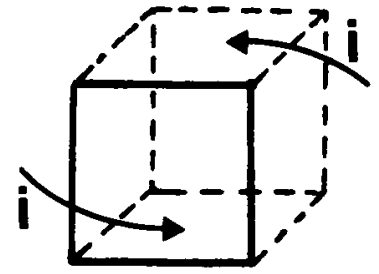

Figure 2. A rotating 3D cube provides relative motion between the front and rear faces as shown by the vectors marked $i$. (If the darker face is in front, the vectors specify counterclockwise rotation. If the dotted face is in front, the vectors specify clockwise rotation.)

Perceptually coupled attributes are aspects of perception that could be independent (i.e., they are different dimensions), but that would normally covary in the case of a real object (Epstein, 1977, 1982; Hochberg, 1956, 1974). For example, for a rotating $3 \mathrm{D}$ cube-like object, the relative distance of the cube's faces and their relative motions in space are normally physically correlated. As is shown in Figure 2, the relative shifting of the front and rear faces of the cube specifies counterclockwise rotation if the lower thicker face is the nearer face. Were the relative distances to the two faces of the cube physically reversed (i.e., if the upper dotted face were the nearer face), the relative shifting of the upper and lower faces would specify clockwise rotation instead. Relative distance and direction of rotation are perceptually coupled as well: If the rear (dotted) face is erroneously perceived to be forward, a cube that is actually rotating in a counterclockwise direction appears to be rotating in a clockwise direction. That is, as the perceived depth organization of the cube changes, so does the perceived direction of rotation (von Hornbostel, 1922), even though there is no change in the physical stimulus.

Other illusions that are coupled to misperceived distance are illusions of size and concomitant motion. With such couplings, one can attempt to separate what subjects perceive from what they report (Gogel, 1980; Gogel \& Tietz, 1974; Peterson, 1986; Peterson \& Hochberg, 1982; Wallach et al., 1953). For example, MacCracken, Gogel, and Blum (1980) showed that errors of perceived size failed to accompany reports of misperceived distance that occurred at the experimenter's posthypnotic suggestion. In addition, Peterson (1986) has used reports about the direction of illusory concomitant motion in a stereogram to provide some assurance that the measured effects of intention (using a procedure similar to the one used with the $3 \mathrm{D}$ cube) were not due to the demand character of the instructions.

Perceptually coupled responses have not previously been used to examine questions of piecemeal perception, however.

\footnotetext{
${ }^{1}$ Since the present paper was submitted, Boselie and Leeuwenberg (1986) have attempted to meet the challenge posed by a $2 \mathrm{D}$ version of a figure similar to the one used in Experiment 2. Their explanations will not do for either the 2D version or for a partially surfaced $3 D$ cube, both of which were used by Peterson and Hochberg (1983). In any case, although they conceded points (a) and (b) above, they made no attempt to deal with them or to predict their consequences. (This point is developed at greater length in Peterson \& Hochberg, 1987.)
} 
Because of the importance of piecemeal perception to perceptual theory, such effects should be replicated under conditions that render a response bias interpretation implausible. ${ }^{2}$ Accordingly, in the experiments reported here, reports about perceptual couplings are used as indirect measures of changes in perceived depth. In Experiments 1 and 2, reports of reversed rotation direction serve as our index of reversed depth. In Experiment 3, reports of illusory concomitant rotation serve the same function. If we can demonstrate that perceptual couplings change in accordance with changes in fixation and with instructed intention within a single moving object, we will have provided reasonably strong evidence (a) that the stimulus is not processed as a single perceptual unit and (b) that the instructions to see one or the other alternative affect what viewers really perceive.

\section{Components of Ambiguity}

In addition, responses about perceptually coupled rotation were used to attempt to separate and measure two different ways in which parts of the same object might contribute to the determinacy or ambiguity of the object's appearance. First, reversals may occur (or may not occur) because the stimulus information per se is differentially ambiguous at different places. We will call this stimulus-based component of ambiguity nonelective instability (I). For example, the biased intersection in Figure 1D may be relatively unambiguous and stable, exerting obligatory stimulus effects in that region, whereas the unbiased point might be ambiguous or unstable (or multistable; Attneave, 1971), in the sense that it exhibits spontaneous or obligatory reversal and is subject to alternating fatigue or satiation processes (Attneave, 1971; Hochberg, 1950; Howard, 1961; Köhler, 1929; Orbach, Ehrlich, \& Vainstein, 1963), regardless of the viewer's intention.

Alternatively, or in addition, an object or feature may show repeated changes in appearance, not because it is subject to spontaneous reversals, but because it is malleable $(M)$ and the viewer entertains alternate readings of it. Such malleability may result from passive fluctuations of attention (Woodworth, 1938) or from some more active process that tends to seek alternatives for long-fixated stimulation through what we might call "perceptual boredom." Moreover, the appearance of an object or feature might fail to change, even though it is malleable, because the appearance may reflect the viewer's intention to continue to perceive the object in only one way.

If these two potential components of ambiguity are mediated by different processes, they may be affected differently by changes in the prevailing stimulus conditions. In order to examine this possibility, the total duration of reversal was partitioned into two components, $M$ and $I$, as follows. For each subject, we obtained the total duration of reversed rotation-direction reports for each intersection across instructions to maintain the cube in one orientation. This was our measure of the overall reversibility $(R)$ of that part of the cube.

$$
R_{\mathrm{i}}=R_{\mathrm{i}}(\mathrm{F})+R_{\mathrm{i}}(\mathrm{B})
$$

where $R_{\mathrm{i}}=$ reports of reversed direction of rotation at a given intersection, $i$, as a function of instruction to try to hold the front $(\mathrm{F})$ or the back $(\mathrm{B})$ wire forward.

Next, any reversed rotation-direction reports made when the viewer tried to hold the front wire forward (i.e., in nonreversed orientation) at a given intersection were attributed to the nonelective instability $(I)$ of that region of the cube. We assumed that an equivalent amount of nonelective reversals occurred when the viewer tried to hold the back wire forward at that intersection. Thus, for a given intersection,

$$
I_{\mathrm{i}}=2\left[R_{\mathrm{i}}(\mathrm{F})\right]
$$

where $I_{\mathrm{i}}$ represents nonelective instability. ${ }^{3}$

Finally, in order to estimate the malleability $(M)$ of a given intersection, we subtracted the durations of reversed depth reported when subjects tried to hold the front wire forward (nonelective reversals) from those reported when subjects tried to hold the back wire forward.

$$
M_{\mathrm{i}}=R_{\mathrm{i}}(\mathrm{B})-R_{\mathrm{i}}(\mathrm{F})
$$

Whenever $M$ is significantly greater than zero, malleability will have been demonstrated as an effect separate from instability. (Because $I$ cannot assume negative values, instability cannot be tested for deviations from zero.) Although $M$ and $I$ are complementary at a given intersection under any one condition, they can vary independently with experimental conditions, inasmuch as reversals can vary with conditions. In the experiments reported here, we examine whether they do indeed differ.

In three experiments, we examined reports about rotation direction for evidence of piecemeal perception. In addition, in order to obtain evidence concerning the question of whether malleability and instability are mediated by different processes, we examined the differences in those measures as a function of differences in viewing condition. In Experiment 1, we found piecemeal effects with monocular viewing. In Experiment 2, we replicated Experiment 1 with binocular viewing and introduced the variable of viewing distance. In Experiment 3, we increased the range of viewing distances and compared monocular and binocular viewing conditions.

In Experiments 1 and 2, the cube rotated and the viewers remained stationary. Active movement might give the viewer more information than passive observation (Gibson, 1966; Michaels \& Carello, 1981). Accordingly, in Experiment 3, we examined whether piecemeal reversal (or indeed any ambiguity whatsoever) occurs in a situation in which the $3 \mathrm{D}$ object was stationary and the viewer was moving.

\footnotetext{
${ }^{2}$ We should note that we will not be able to reject some such possibility with complete certainty. Nowhere in those experiments or in the series of experiments that we describe in this paper can we fully rule out some version of a response bias explanation. We can only strive to render it less predictive and less plausible (Peterson, 1986; Peterson \& Hochberg, 1983).

${ }^{3}$ We assume that the duration of reversals attributable to spontaneous or nonelective instability are equal under both hold instructions. In future attempts to analyze the components of ambiguity, it would be useful to model and test that assumption.
} 


\section{Experiment 1}

\section{Method}

Subjects. A total of 12 college students participated in this experiment in order to fulfill a course requirement. All had vision that was normal or corrected to normal.

Stimuli and apparatus. The stimulus was a $15 \times 15 \times 15 \mathrm{~cm}$ cube, constructed of black 2-mm wire rods. Opaque white paper wrapped around the lower right corner of the cube (see Figure 1E) provided strong depth information, thereby biasing the perception of that intersection (the "biased intersection") toward the orientation of the cube shown in Figure 1C. No additional stimulus bias was added at the other intersection, leaving this intersection relatively "unbiased" (see Figure IE). The cube was mounted on a post and driven by a motor and a cam so that it oscillated at a speed of $23^{\prime} / \mathrm{s}$. The cube changed direction of rotation an average of 8.5 times per trial (range $=7-9$ ). Each half-cycle of oscillation lasted about $4.5 \mathrm{~s}$, and the cube was stationary for about $2.5 \mathrm{~s}$ before changing rotation direction. The motion sequences were constrained so that subjects could always see the two intersections. The largest and smallest distances between the intersections were approximately $3.1^{\circ}$ and $1.9^{\circ}$, respectively. Subjects viewed the cube against a textured plywood background from a distance of $255 \mathrm{~cm}$. At this distance, one face of the cube subtended $3.4^{\circ}$ of visual angle.

Procedure. Using drawings similar to those in Figures 1A, 1B, and $1 \mathrm{C}$, the experimenter showed subjects the two main alternative organizations of an ambiguous cube, noting that these alternatives differ in whether the horizontal or the vertical wire appears nearer at Intersections 1 and 2 of Figure 1A. Next, the corresponding intersections on the 3D wire cube were pointed out. The cube then oscillated through one cycle, and the subjects reported on the direction in which it appeared to move, enabling the experimenter to determine whether each subject used a right- or a left-hand rule to identify clockwise and counterclockwise motion.

Subjects were told that their primary task throughout each trial would be to maintain fixation on either the biased or the unbiased intersection and to keep their attention around the point of fixation. ${ }^{4}$ The labeis "biased" and "unbiased" were not used when speaking to the subjects. Instead, the instructions referred to the "top" and "bottom" fixation point. However, inasmuch as the biased intersection is sometimes the top and sometimes the bottom fixation point, the two intersections will be referred to as the biased and unbiased intersections throughout this article.

As a second task, subjects were to try to maintain either an organization that was consistent with the bias or one that was inconsistent with the bias: At each intersection, they were asked to try to keep either the horizontal or the vertical wire appearing foward. Once the subject became familiar with these instructions, they were abbreviated as "hold horizontal" and "hold vertical," respectively. At the biased intersection, "hold vertical" instructions were consistent with the bias; "hold horizontal" instructions were inconsistent with the bias. At the unbiased intersection, "hold horizontal" instructions were consistent with the bias at the biased intersection; "hold vertical" instructions were inconsistent with that bias. Subjects were asked to try to follow the intention instructions solely by concentrating, rather than by moving their eyes or blinking.

As a third task, subjects were to report continually whether the cube appeared to be stationary, or rotating in a clockwise or a counterclockwise direction. The cube actually oscillated regularly. However, to discourage subjects from resorting to a counting strategy to identify the actual, as opposed to the perceived, direction of rotation, we told them that the cube might appear (a) to switch direction of rotation at any point in its oscillation cycle, (b) to oscillate regularly, stopping at each node, or (c) to continue rotating in the same direction after a stop.
Subjects were tested individually while viewing the cube monocularly and wearing earphones that provided broadband noise to mask apparatus cues. They received fixation and hold instructions before each trial with the stationary cube in view. Then they closed their eyes and the experimenter started the driving motor. On signal, subjects opened their eyes and began a running report about the direction of rotation.

After each of the two 60 -s practice trials, subjects were permitted to ask questions about the nature of their responses, and they were questioned about their success at maintaining fxation and at concentrating on the hold instruction. After these trials, the experimenter summarized the subject's reports to determine whether the recorded and reported data matched. Subjects were told that there were no right or wrong answers; they were to respond about how the motion appeared to them. They were reminded that the fixation and hold instructions were more important than their reports about the direction of rotation. Subjects then participated in eight 60 -s trials, two trials at each fixation point with each hold instruction. The order of trials was counterbalanced within and across subjects.

Seated behind a screen, the experimenter observed the motion of the rod and cam, recording the subjects' verbal responses online and scoring the clockwise and counterclockwise reports as correct or incorrect, depending on the actual rotation. Reports of reversed direction of rotation were scored as incorrect responses. Hence, incorrect responses are our measure of reversed depth organization. The experimenter also recorded the number of half-cycles of oscillation occurring on each trial.

Data scoring. For each trial, we divided the number of half-cycles during which the subject reported the incorrect (i.e., reversed) direction of rotation by the total number of half-cycles that occurred during that trial. For each intersection, for each subject, we calculated the mean proportion of half-cycles on which reversed reports occurred under each instruction, and submitted these data to an analysis of variance (ANOVA).

Next, we derived measures of $R, I$, and $M$, according to Equations 1 , 2 , and 3 presented in the introduction. In order to avoid reporting $R$ proportions greater than 1.0, we divided each $R$ score by 2 to represent the mean reversals at a given intersection (i.e., across instructions), rather than the summed reversals. Consequently, we divided the $I$ and $M$ terms by 2 so that they added up to $R$. We followed the same procedure in Experiments 2 and 3.

\section{Results}

Consistent with a piecemeal account of perceptual organization and with the implications of the Peterson and Hochberg (1983) results, the reports of reversed direction of rotation were a function of both stimulus bias and hold instruction. More reversals occurred at the unbiased intersection (.40) than at the biased intersection $(.16), F(1,11)=29.52, p<.0002$. Results also showed a main effect of hold instruction: At both fixation points, subjects reported more reversals when trying to hold

\footnotetext{
${ }^{4}$ It is important in giving fixation instructions in these experiments that subjects are not encouraged to keep their attention so closely bound to the fixation point that they ignore completely all other parts of the stimulus pattern. It is possible to maintain so narrow a field of attention that one can fit whatever one wishes to the point being fixated and indeed, under these conditions, impressions of depth often disappear entirely or are markedly less impressive than with a freer gaze. At the same time, the subject must make some effort to keep fixation close to the instructed point. For this reason, the importance of fixation was stressed repeatedly and subjects were also asked once or twice to keep their attention around the point of fixation.
} 


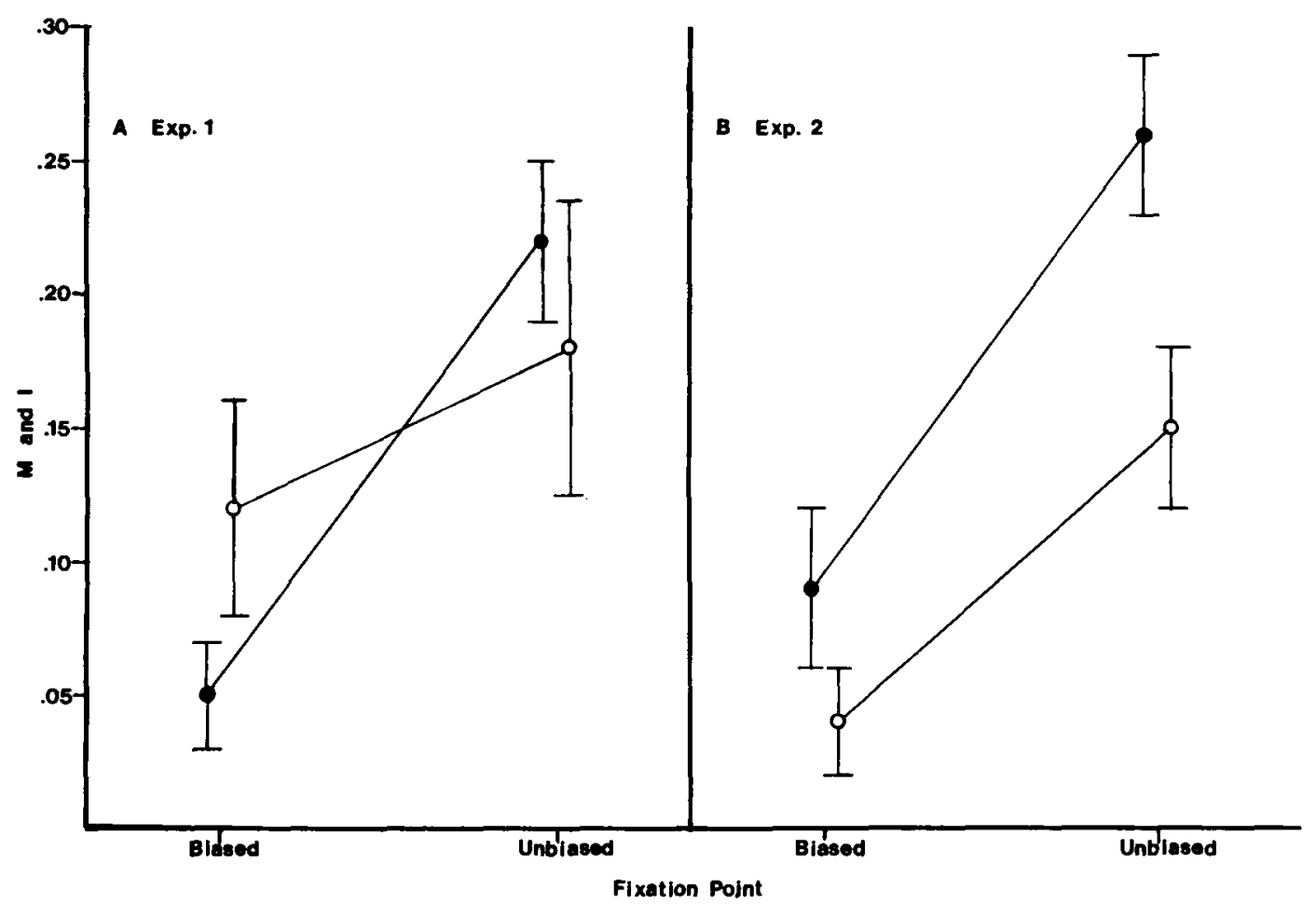

Figure 3. Reversed rotation-direction reports partitioned into those attributable to instability $(I)$ and to malleability $(M)$ as a function of instructed fixation point for Experiment 1 (Panel A) and Experiment 2 (Panel B). (Open circles represent malleability; closed circles, instability. Error bars are standard errors. Note that $R=M+I$.)

forward the back wire rather than the front wire ( .59 vs. .22 , for the unbiased intersection and .28 vs. .05 for the biased intersection), $F(1,11)=13.06, p<.004$.

As is shown in Figure $3 \mathrm{~A}$, the two fixation points differed in instability $(.22$ vs. .05$), F(1,11)=27.78, p<.0003$, but not in malleability $(.18$ vs. .12), $F(1,11)=1.65, p>.20$. This was not because both intersections were equally unmalleable, however: $M$ measures at both intersections were significantly greater than zero, $t(11)=3.18$ and $2.93, p<.01$.

\section{Discussion}

The proportion of reversed rotation-direction responses was higher when viewers were asked to fixate the unbiased intersection rather than the biased intersection, even though these two intersections were parts of a single object and even though the distance between the two intersections was never more than $3.1^{\circ}$. These results were obtained when viewers reported about rotation direction, a variable that is only perceptually coupled to instructed depth. Therefore, the results are not readily attributable to experimenter effect (Orne, 1962). Subjects' reports of the apparent directions of rotation are consistent with the idea that apparent direction of rotation reflects apparent depth, and that apparent depth is affected both by local stimulus information at the attended intersection and by the instructions to perceive one or another organization. These findings replicate those obtained with direct reports about depth (Peterson \&
Hochberg, 1983). Thus, these results suggest that the perceived organization of a single object is not unambiguously determined by stimulus variables such as the local kinetic occlusion available at the biased intersection, nor is it necessarily determined by stimulus-wide information. Instead, these results provide evidence that object organization can occur as a piecemeal process.

The results regarding malleability $(M)$ and instability $(I)$ were rather surprising. As expected, the biased intersection was more stable than the unbiased intersection. The two intersections were equally malleable, however, suggesting that differing degrees of instability may not solely determine differing degrees of malleability. Note that the observers in Experiment 1 viewed the cube monocularly. When the data from Peterson and Hochberg (1983) were reanalyzed into measures of $M$ and $I$, the two fixation points were equally malleable for monocular viewers looking at a 3D cube but not for binocular viewers looking at a 2D cube. (In the latter case, the unbiased intersection was both more unstable and more malleable than the biased intersection.) Thus, the malleability of the biased intersection found here might somehow be specific to monocular viewing. In Experiment 2, we explore this question further by replicating Experiment 1 using binocular viewers.

Three other changes were made in the design of Experiment 2. First, the cube oscillated between clockwise and counterclockwise rotation on a random schedule, rather than on a regu- 
lar schedule as in Experiment 1. This change was intended as a further step toward assuring that the viewers were reporting about perceived, rather than inferred, rotation direction. Second, an attempt was made to improve the sensitivity and reliability of the data. In Experiment 2, viewers' verbal responses were recorded online, together with a video record of the cube's rotation.

In addition, in Experiment 2, observers viewed the cube from two distances: $1 \mathrm{~m}$ and $3 \mathrm{~m}$. We were interested in examining the effects of viewing distance on the degree of independence of the two intersections (i.e., the piecemeal effects) and on the measures of malleability and instability. Because visual angle decreases as distance increases, we were not sure what effects to expect from the distance manipulation. On the one hand, increases in viewing distance ought to decrease the effectiveness of relative depth cues within the object, and therefore, to increase the object's ambiguity. On the other hand, because the angular separation between the two fixation points decreases with increasing distance, the unbiased intersection may come within the effective domain of the kinetic occlusion at the biased intersection with increasing distance. In that case, we would expect the object's ambiguity to decrease with increasing distance.

\section{Experiment 2}

\section{Method}

Subjects. A total of 8 students participated in this experiment for course credit. All had vision that was normal or corrected to normal.

Stimulus and apparatus. In Experiment 2, a piece of white foamcore board was wrapped around the top and the left side of the wire cube used in Experiment 1, creating a solid surface, $5.5 \mathrm{~mm}$ thick, covering the entire top and left sides of the cube. The foam core board biased the cube toward the orientation in Figure 1B. The bottom fixation remained unbiased as in Figure ID. Two 150-W lamps were aligned vertically at a distance of $1 \mathrm{~m}$ from the cube so as to illuminate it from above and below on the right: A 250-W lamp was mounted $1 \mathrm{~m}$ to the left of the cube to illuminate it from below and a 100-W lamp illuminated the top of the cube from $.35 \mathrm{~m}$. The luminance in the region of the biased intersection was $100 \mathrm{ft} l$ and $85 \mathrm{ft} \mathrm{l}$ in the region of the unbiased intersection.

The cube was mounted on a Slo-syn stepper motor $\left(1.8^{\circ} /\right.$ step $)$ driven by a Roger's A6 Timer/Driver Card in an Apple Ile microcomputer. It subtended $8.5^{\circ}$ of visual angle at $1 \mathrm{~m}$ and $2.9^{\circ}$ at $3 \mathrm{~m}$. The cube oscillated randomly between clockwise and counterclockwise rotation at a speed that provided $33 \% / \mathrm{s}$ relative angular velocity between the front and back faces of the cube at $1 \mathrm{~m}$, and $19^{\prime} / \mathrm{s}$ at $3 \mathrm{~m}$. Again, the degree of rotation was restricted so that the 2 intersections remained in view at all times. There were 12 motion segments within the 30-s trial, varying in duration from $1 \mathrm{~s}$ to $4 \mathrm{~s}$, so that the cube moved for $25.6 \mathrm{~s}$ during each trial.

For each trial, both the cube's motion and the subject's verbal responses were simultaneously recorded and later analyzed on a videorecorder (Panasonic NV 8950).

Procedure. The procedure and instructions were those of Experiment 1, with a few exceptions: In Experiment 2, when subjects were given the fixation instructions, they were asked to keep their eyes focused on the front wire at their fixation point. Thus, in this experiment, the subjects' primary task was a fixation-focus task. They were told not to move or defocus their eyes when attempting to hold forward one or the other wire. As in Experiment 1, their secondary task was to hold either the horizontal or the vertical wire forward at the point they were fixating.
Subjects viewed the cube binocularly while seated at both $1 \mathrm{~m}$ and 3 $\mathrm{m}$ from the cube. Half began by viewing the cube from $1 \mathrm{~m}$, half began by viewing the cube from $3 \mathrm{~m}$. Subjects participated in two practice trials, and sixteen 30-s experimental trials, for a total of two experimental trials with each hold instruction at each fixation point at each viewing distance.

Data scoring. The videotaped recordings of each trial were viewed at leisure by a judge who scored the accuracy of rotation-direction reports for each motion segment. Very few reversals of rotation direction occurred within a motion segment (less than $3 \%$ ); when one did occur, each direction was assigned a duration equal to half the motion segment. The data were the proportions of each $25.6 \mathrm{~s}$ of motion on each trial during which reversed rotation direction was reported. Measures of reversal $(R)$, malleability $(M)$, and instability $(I)$ were calculated as in Experiment 1.

The scoring of a random sampling of 30 trials taken from 3 subjects by a second judge, blind to the conditions under which each trial had been obtained, showed virtually perfect reliability $(r=.99)$.

\section{Results}

The results of Experiment 2 replicated the piecemeal effects found in Experiment 1. More reversals $(R)$ occurred at the unbiased interaction than at the biased intersection, $F(1,7)=$ $33.9, p<.0006$. In addition, viewers were more likely to report reversed motion when holding forward the back wire rather than the front wire (.17 vs. .09 for the biased intersection and .56 vs. .26 for the unbiased intersection), $F(1,7)=29.94, p<$ .0009 .

No effects of distance were obtained with any of the measures $(F \mathrm{~s}<1)$.

Unlike Experiment 1, here the two fixation points differed in both instability and malleability. As may be seen in Figure 3B, the unbiased intersection was both more unstable than the biased intersection (.26 vs. .09), $F(1,7)=19.09, p<.003$, and more malleable than the biased intersection (.15 vs. .04$), F(1$, 7 ) $=7.36, p<.03$ (see Figure 3B). In addition, whereas malleability at the unbiased intersection was significantly greater than zero, $t(7)=5.26$, malleability at the biased intersection missed significance, $t(7)=1.76, p<.10$.

\section{Discussion}

As in Experiment 1, viewers who were instructed to look at two different intersections on a single object reported reversals of rotation direction that were a function of both local stimulus cues and intention instruction. The cube used in Experiment 2 rotated on a random schedule and viewers reported about apparent rotation direction, rather than about instructed depth. Thus, we can be reasonably sure that the results are not due to the demand character of the overt instructions used to manipulate intention. We conclude that the phenomenon of piecemeal perception is well supported, and therefore must be addressed by general theories of perception.

The functional separability of the two proposed components of ambiguity is less clear, however. In Experiment 2, which used binocular vision, both malleability and instability were significantly stronger when viewers looked at the unbiased rather than at the biased intersection. Furthermore, the biased intersection did not show reliable malleability effects. In Experiment 1, 
which used monocular vision, the two fixation points differed in nonelective instability, but not in malleability, and both fixation points were malleable. At this point, we do not know whether to attribute these different results to the differential sensitivity of the two experiments or to the different viewing conditions (monocular vs. binocular viewing). Accordingly, in Experiment 3, we compared malleability and instability measures obtained with monocular and binocular viewing.

In addition, viewing distance had no effect in Experiment 2, perhaps because it did not vary over a sufficient range. Accordingly, we used a wider range of viewing distances in Experiment 3.

\section{Experiment 3}

The relative motion of object and observer offered by a moving cube and a stationary observer did not produce unambiguous perception of spatial layout in the first two experiments. Self-produced motion may be of special importance in disambiguating object structure (cf. Gibson, 1966). Therefore, in Experiment 3, we asked whether piecemeal reversal and the effect of intention would be obtained when moving viewers looked at a stationary object. Here, subjects reported about the presence of illusory concomitant rotation rather than about rotation direction. Illusory concomitant rotation is perceptually coupled to misperceived depth in 3D displays (Gogel \& Tietz, 1974). We therefore take these reports to be equivalent to the reports of reversed direction of rotation in Experiments 1 and 2.

\section{Method}

Subjects. A total of 18 paid subjects participated in this experiment. All had vision that was normal or corrected to normal.

Stimulus and apparatus. The cube used here was biased toward the orientation shown in Figure ID. A piece of cpaque white paper was wrapped around the top of the cube, extending halfway across the top and halfway down the left side, so that the biased fixation specifed the organization in which the horizontal wire was forward. The cube subtended $8.5^{\circ}$ at $1 \mathrm{~m}, 4.3^{\circ}$ at $2 \mathrm{~m}$, and $2.2^{\circ}$ at $4 \mathrm{~m}$.

Procedure. The instructions concerning fixation and intention were those of Experiment 2. The procedure was similar, with the following differences. Before the experimental trials, observers viewed a moving cube for a few seconds and labeled the direction of rotation. The cube was then stopped and the observers were told that during the experiment the cube would remain stationary, but that they themselves would move. They were asked to plant their feet at shoulder width and to move from side to side, shifting their weight from one foot to the other to the beat of a metronome. The experimenter demonstrated this lateral motion. While doing so, she pointed out those stationary parts of the room that were alternately occluded or disoccluded by parallax, and explained that, despite the presence of parallactic motion, objectively stationary parts of the room usually appear stationary.

The experimenter then told the subjects that as they viewed the cube while moving laterally, parts of the cube would be successively occluded and disoccluded. She explained that at times they would perceive this occlusion and disocclusion as resulting from their own motion rather than from any motion of the cube. At other times, the occlusion and disocclusion might appear to occur because the cube was moving independently, as it had done earlier when the motor drove the cube. In the latter case, they were to press a hand-held microswitch and keep it depressed as long as the cube appeared to be moving.
During a single experimental session, each subject viewed the cube on two occasions from each of three distances: $1 \mathrm{~m}, 2 \mathrm{~m}$, and $4 \mathrm{~m}$. Each time they viewed the cube at a given distance, they participated in four trials, one trial with each combination of fixation and hold instructions. Half the subjects viewed the cube monocularly, and half viewed the cube binocularly. While giving the instructions, the experimenter was blind as to the subject's viewing condition. In each viewing condition, 3 subjects began by viewing the cube from each distance. Two viewing distance orders were used for each starting position so that a given position was followed by both of the other positions. Within subjects, the order of viewing distance was counterbalanced according to an $\mathrm{ABCCBA}$ design. Fixation instructions were counterbalanced within subjects.

Each subject participated in two practice trials and twenty-four 15-s experimental trials. To ensure that subjects maintained a constant rate and extent of motion, the experimenter viewed each subject against a graduated background on a video monitor. Feedback was given if the subject did not keep pace with the metronome (set at .67 $\mathrm{Hz}$ ) and the trial was rerun later. (Such feedback was rarely necessary after the practice trials.) Subjects' average lateral motion was $40 \mathrm{~cm}$, thereby providing relative angular displacement between the front and rear faces of the cube of approximately $1.76^{\circ} / \mathrm{s}$ at $1 \mathrm{~m}, 31^{\prime} / \mathrm{s}$ at $2 \mathrm{~m}$, and $8 \% / \mathrm{s}$ at $4 \mathrm{~m}$.

\section{Results}

For each subject, the data were the mean proportions of time that the cube appeared to be in motion for each fixation point paired with each hold instruction. At both fixation points, more illusory concomitant motion was reported when subjects tried to hold forward the back wire rather than the front wire $(.21$ vs. .07 for the biased intersection .31 vs. .16 for the unbiased intersection), $F(1,16)=12.01, p<.003$.

Reports of illusory concomitant rotation by moving viewers again support the notion of piecemeal object perception. More reversals occurred at the unbiased intersection $(.23)$ than at the biased intersection $(.14), F(1,16)=37.88, p<.0001$. This difference between biased and unbiased intersections was greater for monocular (.14) than for binocular (.04) subjects, $F(1,16)=8.94, p<.009$. Even though the binocular difference was small, it was significant, $t(8)=1.93, p<.05$. The main effect of viewing condition missed significance, however, $F(1$, 16) $=3.52, p<.08$.

As seen in Figure 4, reversals increased with distance, so that motion was reported $11 \%, 21 \%$, and $21 \%$ of the time for near, medium, and far distances, respectively, $F(2,32)=6.16, p<$ .006 . In a marginal interaction between distance and hold instruction, reversals appeared to peak at $2 \mathrm{~m}$ when viewers tried to hold the front wires forward $(1 \mathrm{~m}=.07,2 \mathrm{~m}=.16,4 \mathrm{~m}=$ .11 ), but to continue to increase between $2 \mathrm{~m}$ and $4 \mathrm{~m}$ when the subjects tried to hold the back wires forward ( $1 \mathrm{~m}=.16,2 \mathrm{~m}=$ $.26,4 \mathrm{~m}=.31), F(2,32)=3.01, p<.06$.

As seen in Figure 5, instability and malleability both varied as a function of distance, but they did not vary in the same way. When viewed from $1 \mathrm{~m}$, the cube was less unstable (.07) than when viewed from $2 \mathrm{~m}(.17)$; when viewed from $4 \mathrm{~m}$, the instability score (.11) did not differ from either the $1-\mathrm{m}$ or the $2-\mathrm{m}$ score, $F(2,32)=3.38, p<.05$. On the other hand, malleability was greater at $4 \mathrm{~m}(.10)$ than at either $1 \mathrm{~m}(.05)$ or $2 \mathrm{~m}(.04)$, $F(2,32)=3.5, p<.04$. Indeed, malleability differed significantly from zero only at $4 \mathrm{~m}$. For monocular subjects, both fixation points were malleable at $4 \mathrm{~m}, t(8)=2.78$ and $t(8)=1.98$, 


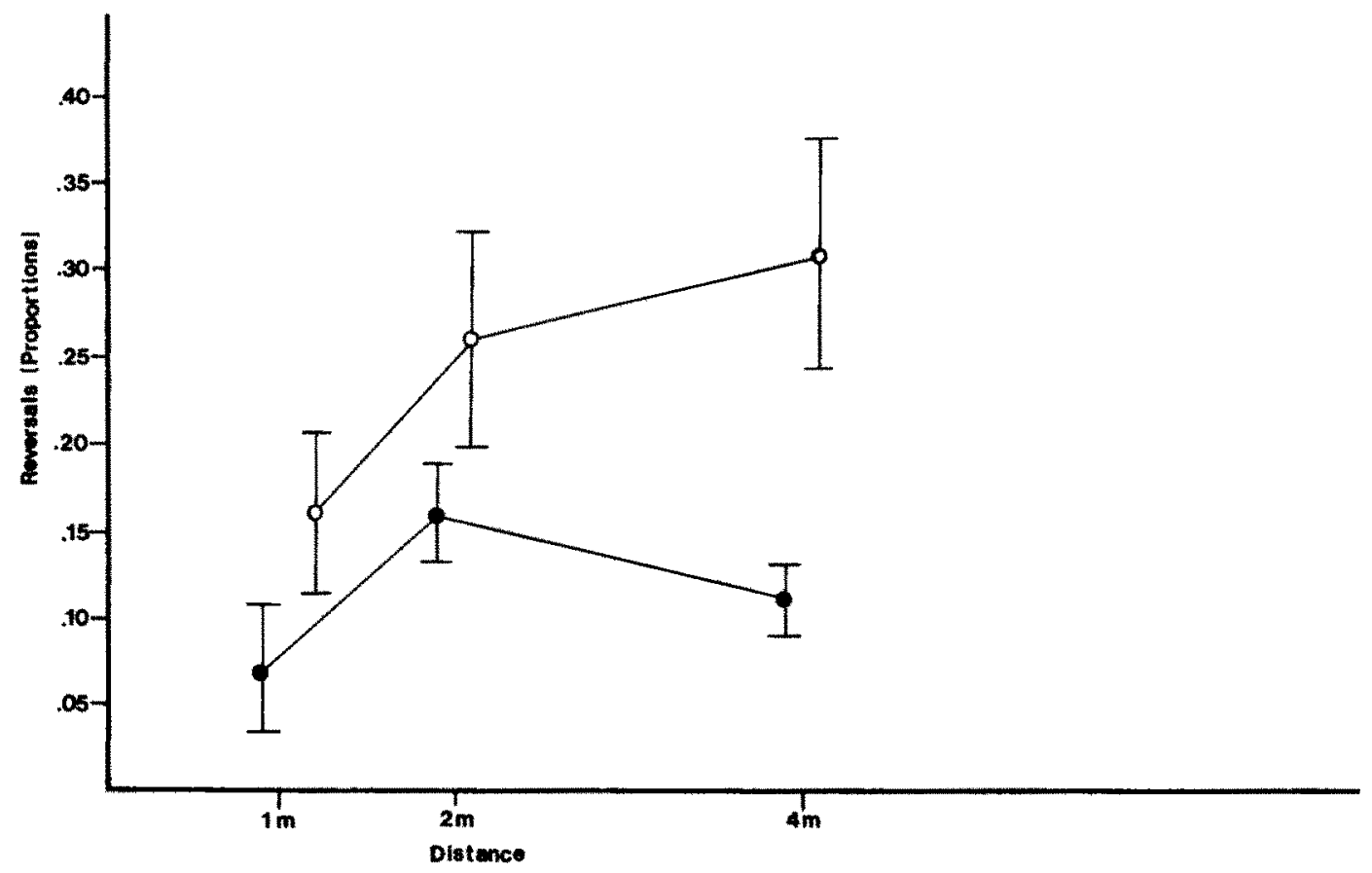

Figure 4. Mean proportions of 15-s trials during which illusory concomitant rotation reports occurred as a function of viewing distance in Experiment 3. (Open circles represent reversals under instructions to bold the back wire forward, closed circles, under hold front instructions.)

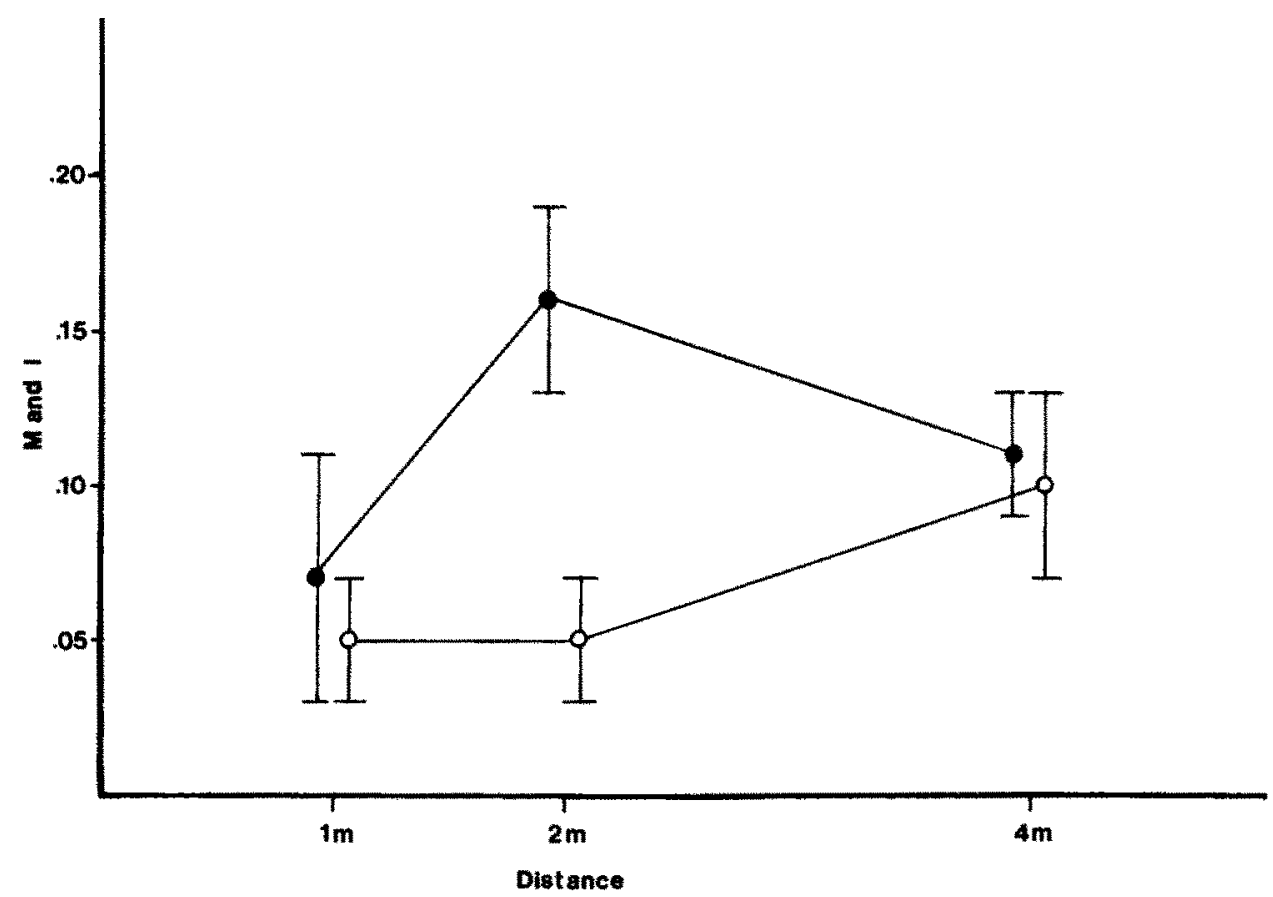

Figure 5. Reversals in Experiment 3 partitioned into those attributable to instability $(I)$ and malleability $(M)$ as a function of viewing distance. (Open circles represent malleability, closed circles represent instability.) 
for fixation at the biased $(.12)$ and unbiased $(.09)$ intersections, respectively. For binocular subjects, only the unbiased intersection was malleable $(.10)$ at $4 \mathrm{~m}, t(8)=2.53$. Malleability at the biased intersection (.08) missed being significantly different from zero, $t(8)=1.44, p<.10$. Differences in malleability between monocular and binocular viewing conditions were not significant, however $(F<1)$, even at $4 \mathrm{~m}$. Despite the apparent differences between malleability and instability as a function of distance, an overall ANOVA examining the differences between malleability and instability as a function of intersection, distance, and viewing condition was not significant, $F(1,16)=1.64$

The unbiased intersection $(.26)$ was more unstable than the biased intersection (.09) with monocular viewing, whereas the binocular viewing, the unbiased and biased intersections were equally stable (biased $=.04$, unbiased $=.05$ ), $F(1,16)=15.70$, $p<.001$.

\section{Discussion}

Despite viewer movement, more reversals occurred at the unbiased intersection than at the biased intersection. Thus, viewer movement did not fully dispel the phenomenon we take as evidence of "piecemeal perception."

Even though self-produced motion per se does not dispel the piecemeal perception effects (as measured by $R$ ), it may dispel some aspects of stimulus ambiguity, especially when observers view a stimulus binocularly. The instability component of $R$ was greater at the unbiased intersection than at the biased intersection with monocular viewing, but not with binocular viewing, and malleability was nonzero only at $4 \mathrm{~m}$. Thus, as depth cues increased, both malleability and instability decreased. The differences in malleability between monocular and binocular viewing conditions in Experiment 3 were not significant, however, so the different results regarding the malleability of the biased intersection that were found in Experiments 1 and 2 may not have been due to the fact that Experiment 1 used monocular viewing and Experiment 2 used binocular viewing.

In Experiment 3, unlike Experiment 2, reversals increased as a function of distance. Both malleability and instability varied with distance, but malleability was manifest only at $4 \mathrm{~m}$, whereas instability was greatest at $2 \mathrm{~m}$. These differences between $M$ and $I$ as a function of distance, although not significant, suggest that malleability and instability may be mediated by separable processes. Perhaps some stimulus information (as yet unspecified) must fall below some level in order to permit viewers' elective intentions to affect perception. Even above that level, however, the reversal of perspective can appear under the impetus of whatever forces provide for nonelective instability. Note that, regardless of the viewing distance at which these effects are visible, they occur despite the static and kinetic depth information at the biased intersection.

\section{Summary and Conclusions}

In the first two experiments, stationary subjects fixated one of two places in a clearly visible, oscillating partially surfaced wire cube. When a rotating cube is incorrectly perceived in re- versed perspective, its direction of rotation should appear reversed as well. In Experiment 3, a laterally moving observer fixated one of two places in a stationary cube. If a stationary cube is perceived in reversed perspective by a moving viewer, illusory concomitant motion should appear.

In all three experiments, the rotations reported by the viewers were consistent with the assumption that the cube was perceived in reversed perspective part of the time. Furthermore, the durations with which it was so perceived $(R)$ were greater when the subjects attended to the relatively uninformative (unbiased) intersection rather than to the informative (biased) intersection, and were greater when the subjects were instructed to try to perceive as nearer a part of the cube that was really farther away. These are the main results, and are consistent with the conclusions of Peterson and Hochberg (1983) that were based on direct reports about depth in a slowly moving cube. In the present experiments, responses were made only in terms of the rotation-direction reversals that are perceptually coupled to reversals of perceived depth, thereby providing reasonable grounds for concluding that viewers really did reverse the depth organization of the 3D cube.

We also attempted to partition the reversal responses into two separable components: instability and malleability. Instability refers to a spontaneous, nonelective, or unintentional reversal in perceived organization, and is estimated from the reversals that occur against viewers' instructed intentions. Malleability refers to the effects of an instructed intention to hold one alternative organization, and is estimated as the reversals that are consistent with viewers' instructed intentions, after estimated instability has been partitioned out. Under most of the present conditions, perception was truly influenced by the viewers' instructed intentions: Measures of malleability were significantly greater than zero when viewers looked at the unbiased intersection (except in the shorter distance conditions in Experiment 3). Instability measures could not be tested against zero, but instability at the unbiased intersection was significantly greater than at the biased intersection in all cases except the binocular group in Experiment 3. Thus, under most of the present conditions, perception was influenced both by the manipulated differences in stimulus bias at the two intersections and by the viewer's intentions. Malleability and instability are distinct arithmetically and they may measure different processes. However, even though there is some suggestion in Experiment 3 that malleability and instability may be affected differently by changes in viewing distance, the differences between the two as a function of distance were not significant. Further research is needed to determine empirically whether the two components of ambiguity are mediated by different processes that are differentially affected by changes in the strength of depth information.

On theoretical grounds alone, neither component of ambiguity can be attributed exclusively to either central or peripheral processes, at this stage of our knowledge. Some writers have attributed reversals to relatively autonomous (albeit central) satiation (Attneave, 1971; Hochberg, 1950; Howard, 1961; Köhler, 1929; Orbach et al., 1963). Others attribute reversal to a more cognitive fluctuation of attention in fitting one or another mental structure (i.e., potential object) to the stimulus display 
(Hochberg, 1970, 1971; Woodworth, 1938). Although a certain degree of "top-down" or nonstimulus influence is necessarily implicated in the malleability effects reported here, there is no reason to assume that the mechanisms are wholly cognitive. Elective changes in perception might be mediated in many ways. They might be mediated cognitively by summoning and fitting one central interpretation rather than another to the stimulus array (Hochberg, 1970), or by attending to one dimension of variation rather than another (Peterson, 1986). More peripheral mechanisms also suggest themselves for this purpose, however. For example, elective changes in perception might be mediated either by directing attention to one site of information rather than another, while maintaining constant fixation (Peterson, 1986), or by the state of accommodation (Hochberg, 1974) or convergence (Peterson, 1986). Convergence changes might in turn provide the consequent illusory concomitant motion (Post \& Leibowitz, 1982) with no additional mediation required. That is, the perceptual coupling manifest with the hold instructions could be mediated by a noncognitive process.

Viewers were instructed to maintain a specific fixation in these experiments. Previous studies have found no eye movement differences during the two opposed hold instructions, within the accuracy of measurement possible with the methods used (Peterson, 1984, 1986). Small, momentary excursions might still differ depending on the hold instructions, however. Moreover, even if fixation were perfectly maintained, we know of course that attention can be directed to regions outside of the fovea (e.g., Posner, Snyder, \& Davidson, 1980). Such changes in the spatial distribution of attention might well account for much or all of the malleability revealed in our research.

These different possibilities shape very different theoretical pictures of the nature of malleability in the perception of objects, and they await experimental separation. Regardless of the mechanisms through which the reversals occur, however, the finding that differing amounts of reversals occur at different fixation points on a single object is quite robust, showing that individual features within a single object can make separate contributions to the perception of three-dimensional layout. These findings cannot be explained by the present forms of those theories in which measurable aspects of stimulus information, taken over an entire object, are offered as the sufficient bases of perceptual organization. If something less than the entire object is to be considered, some principled method of delimiting the effective region must be offered (Peterson \& Hochberg, 1987). Discussions about the limited span of influence of various depth cues, about the selective effects of regional attention to local information or to local depth cues, and about the weighting effects of the viewer's elective intentions, must go hand in hand with attempts to specify stimulus structure.

The present experiments necessarily used fixation instructions, and that may restrict the ecological generality of the findings. In other regards, the conditions would seem to be those of normal perception. If it is argued that such effects occur only with unusual or ecologically unrepresentative stimuli, then we will have to be told with some precision why these real moving objects, or stationary objects viewed by moving observers, fail as tests of perceptual theory, thereby setting the limits of such a theory.

\section{References}

Attneave, F. (1954). Some informational aspects of visual perception. Psychological Review, 61, 183-193.

Attneave, F. (1971). Multistability in perception. Scientific American, 225, 62-71.

Attneave, F. (1972), Representation of physical space. In A. W. Melton \& E. J. Martin (Eds.), Coding processes in human memory (pp. 283306). Washington, DC: Winston.

Boselie, F., \& Leeuwenberg, E. (1986). A test of the minimum principle requires a perceptual coding system. Perception, 15, 331-354.

Buffart, H., Leeuwenberg, E., \& Restle, F. (1981). Coding theory of visual pattern completion. Joumal of Experimental Psychology: Human Perception and Performance, 7, 241-274.

Butler, D. 1. (1982). Predicting the perception of three-dimensional objects from the geometrical information in drawings. Journal of Experimental Psychology: Human Perception and Performance, 8, 674692.

Dinnerstein, D., \& Wertheimer, M. (1957). Some determinants of phenomenal overlapping. American Journal of Psychology: 70, 21-37.

Epstein, W. (1977). Observations concerning the contemporary analysis of constancies. In W. Epstein (Ed.), Stability and constancy in visual perception (pp. 437-447). New York: Wiley.

Epstein, W. (1982). Percept-percept couplings. Perception, 11, 75-83.

Gibson, J. J. (1966). The senses considered as perceptual systems. Boston: Houghton Mifflin.

Gibson, J. J. (1979). The ecological approach to visual perception. Boston: Houghton Miflin.

Gibson, J. J., Kaplan, G. A., Reynolds, H. V., \& Wheeler, K. (1969). The change from visible to invisible: A study of optical transitions. Perception \& Psychophysics, 5, 113-116.

Gogel, W. C. (1980). The sensing of retinal motion. Perception \& Psychophysics, $28,155-163$.

Gogel, W. C., \& Newton, R. E. (1976). An apparatus for indirect measurement of perceived distance. Perceptual and Motor Skills, 43, 295-302.

Gogel, W., \& Tietz, J. (1974). The effect of perceived distance on perceived movement. Perception \& Psychophysics, 16, 70-78.

Hatfield, G., \& Epstein, W. (1985). The status of the minimum principle in the theoretical analysis of visual perception. Psychological Bulletin, 97, 155-186.

Hochberg, J. (1950). Figure-ground reversal as a function of visual satiation. Journal of Experimental Psychology, 40, 682-686.

Hochberg, J. (1956). Perception: Toward the recovery of a definition. Psychological Review, 63, 400-405.

Hochberg, J. (1962). The psychophysics of pictorial perception. AudioVisual Communication Review, $10,22-54$.

Hochberg, J. (1968). In the mind's eye. In R. N. Haber (Ed.), Contemporary theory and research in visual perception (pp. 309-331). New York: Appleton-Century-Crofts.

Hochberg, J. (1970). Attention, organization, and consciousness. In D. I. Mostofsky (Ed.), Attention: Contemporary theory and analysis (pp. 99-124). New York: Appleton-Century-Crofts.

Hochberg, J. (1971). Perception I: Color and shape. In J. W. Kling \& L. A. Riggs, (Eds.), Woodworth \& Scholosberg's experimental psychology (3rd ed.). New York: Holt, Rinehart \& Winston.

Hochberg, J. (1974). Higher-order stimuli and interresponse coupling in the perception of the visual world. In R. B. MacLeod \& H. L. Pick (Eds.), Perception: Essays in honor of James J. Gibson (pp. 17-39). Ithaca, NY: Cornell University Press. 
Hochberg, J. (1981). Levels of perceptual organization. In M. Kubovy \& J. Pomerantz (Eds.), Perceptual organization (pp. 255-278). Hillsdale, NJ: Erlbaum.

Hochberg, J. (1982). How big is a stimulus? In J. Beck (Ed.), Organization and representation in perception (pp. 191-218). Hillsdale, NJ: Erlbaum.

Hochberg, J., \& Brooks, V. (1960). The psychophysics of form: Reversible-perspective drawings of spatial objects. American Journal of Psychology, 73, 337-354.

Hochberg, J., \& MacAlister, E. (1953). A quantitative approach to figural "goodness." Joumal of Experimental Psychology, 46, 361-364.

von Hornbostel, E. M. (1922). Uber optische inversion [On optical inversion]. Psychologische Forschung, 1, 130-156.

Howard, I. P. (1961). An investigation of a satiation process in the reversible perspective of revolving skeletal shapes. Quarterly Journal of Experimental Psychology, 13, 19-33.

Johansson, G. (1982). Visual space perception through motion. In A. H. Wertheim, W. A. Wagenaar, \& H. W. Leibowitz (Eds.), Tutorials on motion perception (pp. 19-39). New York: Plenum.

Kahneman, D., \& Henik, A. (1981). Perceptual organization and attention. In M. Kubovy \& J. R. Pomerantz (Eds.), Perceptual organization (pp. 181-212). Hillsdale, NJ: Erlbaum.

Kahneman, D., \& Treisman, A. M. (1984). Changing views of attention and automaticity. In R. Parasuraman \& D. R. Davies (Eds.), Varieties of attention (pp. 29-62). New York: Academic Press.

Kaplan, G. A. (1969). Kinetic disruption of optical texture: The perception of depth at an edge. Perception and Psychophysics, 6, 193-198.

Köhler, W. (1929). Gestalt psychology: New York: Liveright.

Leeuwenberg, E. (1971). A perceptual coding language for visual and auditory patterns. American Journal of Psychology, 84, 307-349.

MacCracken, P. J., Gogel, W. C., and Blum, G. S. (1980). Effects of post hypnotic suggestion on perceived egocentric distance. Perception, 9, 561-568.

Michaels, C. F., \& Carello, C. (1981). Direct perception. Englewood Cliffs, NJ: Prentice-Hall.

Orbach, J., Ehrlich, D., \& Vainstein, E. (1963). Reversibility of the Necker Cube: III. Effects of interpolation on reversal rate of the cube presented repetitively. Perceptual and Motor Skills, 17, 459-464.

Orne, M. T. (1962). On the social psychology of the psychological exper- iment: With particular reference to demand characteristics and their implications. American Psychologist, 17, 776-783.

Penrose, L., \& Penrose, R. (1958). Impossible objects: A special type of visual illusion. British Journal of Psychology, 49, 31-33.

Peterson, M. A. (1984). Measures of selective components in perceptual organization. Unpublished doctoral dissertation, Columbia University, New York.

Peterson, M. A. (1986). Illusory concomitant motion in ambiguous stereograms: Evidence for nonstimulus contributions to perceptual organization. Journal of Experimental Psychology: Human Perception and Performance, 12, 50-60.

Peterson, M. A., \& Hochberg, J. (1982). Attention and local depth cues affect the perception of (and not merely the reports about) real objects. Proceedings of the Eastern Psychological Association, 53rd Annual Meeting (p. 97). (Abstract)

Peterson, M. A., \& Hochberg, J. (1983). Opposed-set measurement procedure: A quantitative analysis of the role of local cues and intention in form perception. Journal of Experimental Psychology: Human Perception and Pefformance, 9, 183-193.

Peterson, M. A., \& Hochberg, J. (1987). Necessary considerations for a theory of form perception: A reply to Boselie and Leeuwenberg. Manuscript submitted for publication.

Pomerantz, J. R., \& Kubovy, M. (1986). Theoretical approaches to perceptual organization. In K. R. Boff, L. Kaufman, \& J. P. Thomas (Eds.), Handbook of perception and human performance (Vol. 2, pp. 36: 1-46). New York: John Wiley \& Sons.

Posner, M. I., Snyder, C. R., \& Davidson, B. J. (1980). Attention and the detection of signals. Journal of Experimental Psychology: General, 109, 160-174.

Post, R. B., \& Leibowitz, H. W. (1982). The effect of convergence on the vestibulo-ocular reflex and implications for perceived movement. Vision Research, 22, 461-465.

Wallach, H., O'Connell, D. N., \& Neisser, U. (1953). The memory effect of visual perception of three-dimensional form. Journal of Experimental Psychology, 45, 360-368.

Woodworth, R. S. (1938). Experimental psychology. New York: Holt.

Received September 23, 1986

Revision received March 18, 1987 Accepted June 17, 1987 